\title{
Putting pain assessment into practice: Why is it so painful?
}

\author{
Linda S Franck PhD RN RGN RSCN FRCPCH FAAN ${ }^{1,2}$, Elizabeth Bruce MSc BSc (Hons) RGN RSCN ${ }^{2}$
}

\author{
LS Franck, E Bruce. Putting pain assessment into practice: Why is \\ it so painful? Pain Res Manage 2009;14(1):13-20.
}

\begin{abstract}
OBJECTIVE: To explore some of the reasons for poor compliance with the use of standardized pain assessment tools in clinical practice, despite numerous guidelines and standards mandating their use.

METHODS: First, a review of research and clinical audit literature on the effects of standardized pain assessment tools on patient or process outcomes was conducted, and findings were critiqued. Second, a synthesis of recent literature on the biopsychosocial mechanisms of human detection and recognition of pain in others was presented. Third, the implications for pain assessment in pediatric clinical settings were discussed.

RESULTS: There is a lack of good-quality evidence for the efficacy, effectiveness or cost-benefit of standardized pain assessment tools in relation to pediatric patient or process outcomes. Research suggests that there may be greater variability than previously appreciated in the ability and motivation of humans when assessing pain in others. It remains unknown whether pain detection skills or motivation to relieve pain in others can be improved or overcome by standardized methods of pain assessment.

DISCUSSION: Further research is needed to understand the intra- and interpersonal dynamics in clinical assessment of pain in children and to test alternative means of achieving diagnosis and treatment of pain. Until this evidence is available, guidelines recommending standardized pain assessment must be clearly labelled as being based on principles or evidence from other fields of practice, and avoid implying that they are 'evidence based'.
\end{abstract}

Key Words: Children; Clinical practice guidelines; Pain recognition; Standardized pain assessment tools

\section{Pourquoi est-il si «pénible » d'évaluer la douleur dans la pratique?}

OBJECTIF : Explorer certains facteurs susceptibles d'expliquer la piètre conformité aux pourtant si nombreuses recommandations et normes relatives à l'emploi d'outils standardisés d'évaluation de la douleur dans la pratique clinique.

MÉTHODES : Les auteurs ont commencé par consulter la littérature publiée sur la recherche et les audits cliniques ayant pour objet les résultats des outils standardisés d'évaluation de la douleur chez les patients ou les résultats des interventions; ils ont ensuite analysé les conclusions de façon critique. L'étape suivante a été une synthèse de la littérature récente sur les mécanismes biopsychosociaux de détection et de reconnaissance de la douleur chez l'être humain. Troisièmement, les auteurs ont mesuré les implications de l'évaluation de la douleur dans des contextes cliniques pédiatriques.

RÉSULTATS : On déplore l'absence de preuves de bonne qualité sur l'efficacité, l'efficience ou le rapport coût:bénéfices des outils standardisés d'évaluation de la douleur ou des effets des interventions chez les patients pédiatriques. Il ressort de la recherche que les capacités et la motivation à l'égard de l'évaluation de la douleur chez l'être humain seraient peut-être plus variables qu'on l'a d'abord cru. On ignore si, par des méthodes standardisées d'évaluation de la douleur, il est possible d'améliorer les capacités ou la motivation à l'égard de la détection de la douleur chez autrui pour la soulager ou de surmonter les obstacles à ce chapitre.

DISCUSSION : Il faudra approfondir la recherche pour comprendre les dynamiques intra- et interpersonnelles propres à l'évaluation clinique de la douleur chez les enfants et pour tester des solutions de rechange en vue de diagnostiquer et de traiter efficacement la douleur. Tant qu'on ne disposera pas de preuves tirées de telles recherches, il faudra clairement indiquer que les lignes directrices recommandant l'emploi d'outils standardisés d'évaluation de la douleur se fondent sur des principes ou des preuves provenant d'autres champs de pratique et éviter de dire qu'elles sont "basées sur des preuves ».

guidelines, educating and auditing clinicians, and generally lamenting the poor integration of pediatric pain assessment in children's health care, perhaps it is time for those who work in the field to pause and reflect on whether the problem is more than simply one of translating research into practice. There may be something fundamentally flawed with the evidence base for the clinical practice of pain assessment. We must be prepared to consider the possibility that time and expensive organizational resources have been misdirected.

The critical question to be addressed if pain assessment is to be fully integrated into the caregiving routines for all children receiving hospital care is whether the use of structured pain both in pediatrics and adult care. After over 30 years of developing and validating measures, producing standards and

\footnotetext{
${ }^{1}$ Institute of Child Health, University College London; ${ }^{2}$ Great Ormond Street Hospital for Children NHS Trust, London, United Kingdom Correspondence: Professor Linda S Franck, Institute of Child Health and Great Ormond Street Hospital for Children NHS Trust, Level 7 , Paul O'Gorman Building, Great Ormond Street, London WC1N 3JH, United Kingdom. Telephone 44-0-20-7829-7822,

fax 44-0-20-7829-8602, e-mail l.franck@ich.ucl.ac.uk
} 
assessments with validated pain assessment tools in clinical practice improves patient outcomes in clinical trials (efficacy) and real-world practice (effectiveness). However, one may argue that it is difficult or impossible to separate the effect of structured assessment from the effects of pain treatment on patient outcomes. Pain assessment may be better evaluated by intermediate or process outcomes that enable clinicians to give more effective treatment. Therefore, while one may not observe a direct effect of assessment on pain relief, one may see, for example, greater documentation of pain, which then facilitates more effective treatment and, in turn, improves patient outcome (2). Thus, the evidence for pain assessment effectiveness may be imbedded in studies of pain management interventions.

In the first part of the paper, we present the findings of a critical literature review on the effectiveness of standardized pain assessment tools on improving clinical outcomes for hospitalized pediatric patients. The review demonstrates a weak evidence base underpinning current clinical practice guideline recommendations for use of standardized pain assessment tools. In the second part, we challenge the assumptions regarding the need for standardized pain assessment tools by reviewing recent research evidence on the biopsychosocial mechanisms of pain recognition in humans and suggest that the selection or training of individuals who are sensitive to detecting pain in others could be more effective in improving pain treatment. We propose the need for randomized clinical trials to compare the efficacy of standardized pain assessment tools or to improve pain detection capabilities of health care professionals. We further propose that, in the absence of direct evidence of effectiveness of standardized pain assessment tools on patient or process outcomes, guidelines recommending standardized pain assessment be clearly labelled as being based on principles or evidence from other fields of practice rather than being called 'evidence-based' standards.

\section{DOES PAIN ASSESSMENT IMPROVE PEDIATRIC PATIENT OUTCOMES? A CRITICAL REVIEW}

\section{Search strategy}

A literature search was performed in the MEDLINE and CINAHL databases for studies published between 1990 and 2008 using the following search terms: pain and measur* or scale*, or pain measurement/methods and validity, reliability, sensitivity, specificity, predictive value, positive predictive value or reproducibility of results, or pain and documentation. The search criteria terms were limited to children, or paediatric* or pediatric*. The study types were limited to clinical trial, comparative study, controlled clinical trial, evaluation study, metaanalysis, multicentre study, randomized controlled trial, review or validation study. PubMed, the Cochrane Library and article reference lists were also searched. The titles and abstracts were hand-searched by both authors to determine whether studies met the inclusion criteria to further reduce the sample to clinical audit or research studies investigating the effects of implementing pain assessment tools or protocols on pediatric patient outcomes in an acute care hospital setting. Studies were excluded if nurses were not involved in the assessment process, because nurses are usually the health care professionals responsible for pain assessment in acute care pediatric settings. Studies were also excluded if the pain assessment method was not evaluated (ie, described only), or if they were case studies.
Studies that implemented education interventions and/or new treatment guidelines were included if the implementation of a standardized pain assessment tool or protocol was clearly identified as a component of the intervention. Studies were included if they investigated any patient clinical outcome, such as pain relief, analgesic consumption or recovery from illness. Studies were excluded if they evaluated outcomes solely related to the clinician (eg, nurses' knowledge or attitudes). All relevant studies were reviewed and abstracted by both authors, using the criteria from the Cochrane Effective Practice and Organisation of Care Group Data Collection Checklist as a guide, although all papers were included even if they did not meet the Cochrane Effective Practice and Organisation of Care Group criteria (7). Both reviewers independently assessed the quality of each study, resolving any differences by discussion.

\section{Results}

The Cochrane Library search revealed no relevant reviews on the topic. Three recent systematic reviews (8-10) of standardized pain assessment tools were found, but these were excluded because they examined the performance of tools in measuring pain, but did not evaluate the effectiveness of the tools in achieving patient or process outcomes. The search revealed 605 abstracts, of which many were irrelevant to the topic because the subject of investigation was development of an assessment tool or evaluation of a specific pain-relieving intervention. One study (11) of physician-only assessment of pain in the emergency department was excluded. Two clinical audits $(12,13)$ were subsequently excluded because of insufficient information regarding audit methods and results, or lack of baseline for comparison. The final sample consisted of 14 studies (Table 1). All articles were in English, apart from one Finnish paper with an English abstract. Only four studies (14-17) were randomized controlled trials (RCTs) and the remaining 10 studies were before-and-after (18-25) or timeseries $(26,27)$ comparisons.

Only four studies $(15,17,18,24)$ investigated the introduction of standardized pain assessment tools alone, without any change to pain treatment. Various self-report and observational pain tools were used, and several tools were often used within one project. Two studies $(14,25)$ investigated the effects of standardized pain assessment through introduction of a new pain assessment and treatment documentation sheet along with the use of standardized pain assessment tools. In the remaining studies, the introduction of the various pain assessment tools formed part of a combined intervention that included introduction of pain education and/or pain treatment guidelines or protocols $(16,19-23,26,27)$.

The quality of reporting varied considerably, and all of the studies had methodological problems. The most common and serious problems were a lack of detail provided on the sample selection, intervention and method of evaluation; poor compliance with the intervention and lack of quality checks (including inter-rater reliability with use of the tools); and long duration between pre- and postintervention evaluation periods. In many of the studies, the sample sizes were small, nonrandom and lacked rigour in the statistical analyses.

The effect of pain assessment on patient outcomes: The effect of standardized pain assessment tools, alone or in combination with other interventions, on patient outcomes was measured in 
TABLE 1

Studies of the effect of standardized pain assessment tools on pediatric patient or process outcomes

Author

(reference),

country Setting, sample

Design

Interventions*

Patient or process outcomes

Methodological issues

Boughton et al Single pediatric ward,

(18), USA

general hospital

Convenience sample, postoperative

( $n=50$ pre, $n=36$ post)

Age: 5 to 17 years

Buchanan 2 pediatric wards,

et al (19), general hospital

Saudi Arabia Convenience sample, high risk for pain $(\mathrm{n}=40$ pre, $\mathrm{n}=40$ post- 1 ; $\mathrm{n}=40$ post-2)

Age: 0 to 14 years

\author{
Ellis et al (20), 5 pediatric wards, \\ Canada \\ general hospital \\ Convenience sample \\ ( $n=75$ pre, $n=44$ \\ post- $1, \mathrm{n}=50$ post- 2 ) \\ Age: Unspecified
}

Falanga et al Single pediatric ward,

(21), Canada general hospital

Convenience sample (pre), all eligible (post), trauma, surgery, painful medical conditions ( $\mathrm{n}=56$ pre, $\mathrm{n}=56$ post)

Age: 5 to 17 years

Franck et al All wards, children's

(15), UK hospital

Randomized, postoperative sample ( $\mathrm{n}=49$ control; $\mathrm{n}=37$ intervention)

Age: 6 to 12 years

Furdon et al NICU, children's hospital

(22), USA

All ventilated neonates after abdominal surgery ( $n=14$ pre, $n=15$ post)
Pre/post comparison T: WBFPS

(clinical audit)

Pre sample obtained

by retrospective

chart review (interval

unspecified)

Pre/post comparison E: CQI

(clinical audit) T: PPAT

3 times over 8 months D: Pain

assessment and

treatment chart

G: Pain

management

guideline

Pre/post comparison

(clinical audit)

3 times (interval

unspecified)$$
\text { , }
$$

E: Inservice and coaching

T: Pain assessment scales (not specified)

D: New documentation

G: Standard care plan for pain management graphic scale,

Pain treatment

\section{Pre/post comparison}

(1 year after pre)

RCT

$$
\begin{aligned}
& \text { Pre/post comparison } \\
& \text { (clinical audit) } \\
& \text { Samples obtained by } \\
& \text { retrospective chart } \\
& \text { review (1 year before } \\
& \text { and } 1 \text { year after } \\
& \text { 2-year intervention) }
\end{aligned}
$$

E: Inservice

T: Colour/word VAS algorithm held paper version

\section{E: CQI}

T: NIPS plan for pain management
Patient: No difference in analgesic efficacy, difference in progress of ambulation or length of stay

Process: No difference in amount of analgesia received

Process: No difference in use of nonpharmacological interventions or in amount of analgesia received (continued 90\% analgesia treatment); increased assessment, re-evaluation and documentation to near $100 \%$ at 3 months; some aspects decreased to approximately $70 \%$ at 6 months; some differences based on patient age, sex

T: WBFPS child Patient: Child/parent satisfaction increased temporary tattoo in intervention group

versus nurse- Process: No difference in pain assessment documentation or analgesia given from 1.5 to 2.1 points; no difference in side effects

Process: Nonopioid and combined analgesia use increased

G: Standard care
Hamers et al Single pediatric ward, (23), The Netherlands

\section{general hospital}

Randomized, postoperative sample tonsillectomy \pm adenoidectomy $(n=42$ control, $n=41$ intervention) Age: 3 to 6 years and 7 to 12 years

\author{
Pre/post (nested within T: Various \\ G: RCT of \\ acetaminophen \pm surgery \\ fentanyl or \\ placebo \\ Patient: No differences in pain scores,
} use of other pain control methods; no

Patient: Pain intensity decreased post

Not reported: Amount of teaching provided, uptake, inter-rater reliability, where assessment was documented or if treatment protocols were used

Not reported: Amount of teaching provided, uptake, inter-rater reliability

Some description of education methods and duration Not reported: Uptake or inter-rater reliability; random selection of $10 \%$ of all available records (post-1), patients likely to have pain (post-2)

Not reported: Amount of teaching provided, uptake, inter-rater reliability

Confounded by strong effect of pain service and/or PCA on amount of documentation and treatment; underpowered

Not reported: Amount of teaching provided, uptake, inter-rater reliability

No statistical analysis of findings effectiveness; increased patients receiving analgesia and continuous infusion; decreased use of bolus morphine and overall consumption

Standardized pain assessment tools introduced to both groups midway in the trial; may have been underpowered 
TABLE 1 - CONTINUED

Studies of the effect of standardized pain assessment tools on pediatric patient or process outcomes

\begin{tabular}{|c|c|}
\hline $\begin{array}{l}\text { Author } \\
\text { (reference), } \\
\text { country }\end{array}$ & Setting, sample \\
\hline \multirow[t]{3}{*}{$\begin{array}{l}\text { Johnston et al } \\
\text { (16), Canada }\end{array}$} & $\begin{array}{l}\text { Multiple (unspecified) } \\
\text { pediatric wards, } \\
6 \text { hospitals ( } 3 \text { children's } \\
\text { hospitals) }\end{array}$ \\
\hline & $\begin{array}{l}\text { Hospitals randomized, } \\
\text { patient chart sampling } \\
\text { unspecified ( } n=464, \\
306 \text { [985 charts] pre, } \\
158 \text { [617 charts] post) }\end{array}$ \\
\hline & Age: Unspecified \\
\hline
\end{tabular}

Jordan-Marsh Multiple (unspecified) et al (26), pediatric wards, general USA

$$
\text { hospital }
$$

Random selection of $10 \%$ of patient charts (unspecified)

at each time point

Age: Unspecified

Joyce et a

(25), USA

Single surgical ward,

children's hospital

Prospective review of all patient charts for $48 \mathrm{~h}$, all children admitted at each time point ( $n=20$ pre, $n=22$ post)

Age: 3 to 6 years

Oakes et al Entire children's cancer

(27), USA hospital

All patient charts for 87

$24 \mathrm{~h}$ periods $(n=2478)$

Age: Newborn to young

adult
Design

Cluster RCT

Pre/post comparison

Prospective review of

patient charts

2 weeks pre and

post

(and (interval unspecified)

\section{Interventions* Patient or process outcomes}

E: 1-to-1 coaching Process: Increased documentation of pain Not reported: Details of

using think aloud assessment (10\% to $40 \%)$ and type and amount of

process nonpharmacological treatments (11\%) for coaching received per

T: Encouraged to

use standardized

pain assessment

tools (various)

G: Encouraged to

implement

evidence-based

pain management

and guidelines

(some sites only)

\section{Pre/post (serial) and after 2-phase,}

comparisons during T: Poker Chip Tool

3-year intervention

G: Clinical practice

guideline; pain management

rounds

Pre/post comparisons
(6 weeks after
intervention)

T: Various

D: Pain

assessment

and management

flowsheet

intervention hospitals but not control sites; nurse

intervention sites; decrease in analgesia at and retention in the

control sites

study

Large variability across sites not accounted for in analysis and may confound findings

Process: Increased documentation of

Not reported: Amount of assessment (30\%) and effectiveness (9\%); teaching provided increased amount of analgesia (87\%) and uptake, inter-rater change in types of drugs given

reliability

Insufficient details about samples at all measurement points

Patient: No difference in parent satisfaction

Process: Decreased pain assessment or management documentation; no difference in documentation of analgesia; increased

Not reported: How intervention was

implemented

High levels of parent satisfaction before no difference in amount of analgesia at Difficulties in recruitment documentation of nonpharmacological intervention intervention

Pre/post comparisons (quarterly over 6 years)

E: Coaching, CQI Patient: Decreased number of patients

T: Pain assessment with pain scores $\geq 5(9 \%)$

scales (NRS, Process: Increased documentation of WBFPS, FLACC) assessment (13\%)

D: New

documentation

G: Pain management protocol

RCT

T: VAS, CHEOPS

D: Pain assess-

ment and

management flowsheet ( $n=20$ intervention, $\mathrm{n}=23$ control)

Age: 1.5 to 12 years

\author{
Pre/post comparison \\ (1-year interval)

\section{E: CQI \\ T: CHEOPS, WBFPS, VAS \\ $\mathrm{G}$ : Pain assess-} \\ ment protocol
}

Patient: Decreased mean pain scores by

\section{6 points}

Process: Increased pain assessment in intervention group (every $4 \mathrm{~h}$ versus every $6 \mathrm{~h}$ ); increased ratings by parents and children in intervention group; increased analgesia administration (25\%) in intervention group; no difference in length of stay

Patient: Increased patient satisfaction with pain tools; no difference in pain or mood ratings or effectiveness of analgesia

Process: Increased documentation of assessments; use of nondrug interventions and speed of staff response to complaints of pain

\section{Not reported: Amount of teaching provided, uptake, inter-rater reliability \\ No statistical analysis of findings \\ Not reported: Amount of teaching provided, uptake, inter-rater reliability teaching provided, uptake, inter-rater reliability \\ Not reported: Amount of}

10 studies. Only three of these $(14,15,17)$ were RCTs. The remainder used before-and-after designs $(18,20-24)$ and a timeseries design (27). Sample sizes varied from 31 to 2478 , and interventions were implemented in diverse settings from single wards to entire hospitals.
The effect of the interventions on pain intensity was examined in six studies. Of these, two found a significant reduction in pain intensity for children when a standardized pain assessment tool was used alone (17) or in combination with a specific pain documentation flowsheet (14) compared with the usual care (ie, 
TABLE 1 - CONTINUED

Studies of the effect of standardized pain assessment tools on pediatric patient or process outcomes

\begin{tabular}{|c|c|c|c|c|c|}
\hline $\begin{array}{l}\text { Author } \\
\text { (reference), } \\
\text { country }\end{array}$ & Setting, sample & Design & Interventions* & Patient or process outcomes & Methodological issues \\
\hline $\begin{array}{l}\text { Vihunen and } \\
\text { Sihvonen } \\
\text { (17), Finland }\end{array}$ & $\begin{array}{l}2 \text { pediatric wards, general } \\
\text { hospital } \\
\text { Sampling unspecified, } \\
\text { postoperative tonsillectomy } \\
\text { ( } \mathrm{n}=80 \text { pre, } \mathrm{n}=80 \text { post, } \\
40 \text { each ward) } \\
\text { Age: } 3 \text { to } 8 \text { vears }\end{array}$ & $\begin{array}{l}\text { RCT: Ward } \\
\text { randomized } \\
y\end{array}$ & T: WBFPS & $\begin{array}{l}\text { Patient: Decreased pain on intervention } \\
\text { ward }(P<0.05) \text {; increased parent } \\
\text { satisfaction }\end{array}$ & $\begin{array}{l}\text { Methods of implementa- } \\
\text { tion not detailed in } \\
\text { English abstract; } \\
\text { differences in pain } \\
\text { management not } \\
\text { controlled between } \\
2 \text { wards }\end{array}$ \\
\hline
\end{tabular}

*E Education; D New pain documentation system; G Pain treatment guideline; T New assessment tool. CHEOPS Children's Hospital of Eastern Ontario Pain Scale; CQI Continuous quality improvement; FLACC Faces, Legs, Activity, Cry, Consolability scale; NICU Neonatal intensive care unit; NIPS Neonate and Infant Pain Score; NRS Numeric rating scale; PCA Patient-controlled analgesia; Post Postintervention; PPAT Pediatric Pain Assessment Tool; Pre Preintervention; RCT Randomized controlled trial; VAS Visual analogue scale; WBFPS Wong-Baker FACES Pain Scale

no standardized pain assessment tool or conventional charting, respectively). Two studies $(21,27)$ found a reduction in pain intensity after implementation of combined pain assessment and pain management interventions, whereas the other studies found no difference in pain intensity after implementation of a standardized pain assessment alone (18) or in combination with pain management guidelines (23). However, in one of these studies (23), only $35 \%$ of pain assessments had been completed, which may have been a confounding factor. Only one study (22) found any improvement in other clinical parameters measured, such as length of stay and side effects, after implementation of standardized pain assessment and treatment protocol in a neonatal intensive care unit. Others (18) found no effect on length of stay, progress to ambulation or effectiveness of analgesia.

Patient or parent satisfaction was examined in four studies, and three found that parent or child satisfaction (eg, perception of the helpfulness of the tools, speed of nurse response and use of nonpharmacological interventions) increased with implementation of standardized pain assessment alone $(15,17,24)$, whereas no change was found in one study (25) in which parent satisfaction was high in the preimplementation period.

The effect of pain assessment on process outcomes: The frequency of pain assessment documentation improved in six studies $(14,16,19,20,24,27)$, but not in four others $(15,18,23,25)$. However, in two studies $(19,20)$ the improvement declined significantly within six to eight months after implementation. Documentation of nonpharmacological interventions was found to improve in five studies $(14,16,20,24,25)$, and three studies found an increase in the amount of analgesia patients received postintervention (21) or on the experimental ward $(14,23)$. However, three studies found no difference in analgesia use $(15,18,25)$.

Summary of the research to date and unanswered questions: The findings suggesting possible benefits of standardized pain assessment tools by nurses for patient (reducing pain scores, and increasing patient and parent satisfaction) or process (increasing pain assessment, treatment documentation and pain treatment delivery) outcomes must be interpreted with extreme caution due to the preponderance of combined interventions and multiple major methodological problems. In all the before-and-after studies, the observed changes may be due to maturation effects and not the intervention itself. Few studies examined sustainability over time and those that did showed conflicting results. It remains unclear whether these findings are generalizable to other health care professionals (eg, physicians, therapists and psychologists) using pain assessment tools because the studies were either restricted to nursing pain assessment, or did not give sufficient detail regarding the composition of the multidisciplinary team or did not compare results between disciplines.

It is also important to highlight the outcomes that have not been explored in any of the studies. For example, no study compared one method of standardized pain assessment with another in relation to patient or process outcomes. No study evaluated the cost of implementing standardized pain assessment tools, or compared the cost-benefit of different methods. Functional patient outcomes were also largely absent from the investigations. No study has examined potential adverse effects of standardized pain assessment tools, such as analgesic overuse or increase in clinical team conflict, or investigated more generalized positive effects such as enhanced coping or improved psychological well-being.

\section{DETECTION AND RECOGNITION OF PAIN IN OTHERS: BIOPSYCHOSOCIAL MECHANISMS AND IMPLICATIONS FOR PAIN ASSESSMENT IN CLINICAL SETTINGS}

All of the pain assessment tools identified in the above systematic review use one of three approaches: asking the verbal child to give a self-report rating of his or her pain intensity using a standard scale and method of questioning; asking the observer to code behavioural, and sometimes verbal, cues in accordance with a predetermined standard rating system; or asking the observer to give a rating of his or her global impression of pain intensity without specifying the specific cues to use. Three key assumptions of these approaches to pain assessment tools are that human beings are capable of reliably and objectively transforming the verbal or behavioural signals expressed in a variety of ways by another person into an objective representation of the signals; a person is motivated to act on recognition of another person's pain; and pain detection and representation 
skills can be improved or motivation to act can be enhanced by the use of standardized methods of assessment. Research over the past decade has shed some light on the neurological basis of pain recognition and the evolutionary factors that may influence the capabilities and motivations of one person to detect pain in another and to act on it. These findings may explain why the use of standardized pain assessment tools may not, in fact, improve pain recognition and treatment in clinical settings, and the need for alternative approaches is suggested.

\section{The mechanisms of pain recognition}

Advances in brain imaging have led to localization of the areas of the brain that receive and process signals about pain experienced by others. The amygdala, prefrontal cortex, insula, anterior cingulate cortex and somatosensory cortices are all involved in recognition of emotion in facial expression, with the amygdala and anterior cingulate cortex playing a prominent role in the recognition of pain. Functional magnetic resonance studies (28) show that similar areas of the brain are activated in response to actual pain experienced, as well as to pain observed in others. Empathic recognition, defined as the 'sense of knowing' the experience of another, is influenced by the observer's dispositional traits and experiences, the observed person's behaviours (particularly facial and verbal expression), sex and contextual factors (eg, relationship or affinity between the observer and the observed).

Accurate detection of the emotional content of facial expression, including pain expression, and empathetic responses can be impaired due to neurological immaturity (29), aging (30), damage (31) or congenital defects (32). Natural and drug-induced mood states also interfere with accurate detection of emotional expression in others (33-35). In contrast, pain catastrophizing traits may enhance detection of pain expression in others (36). Finally, the social role of the observer may affect pain recognition ability. Health care professionals have been found to be less accurate in identifying pain expression in other adults $(37)$ or in infants $(38,39)$ compared with nonprofessionals or parents.

\section{Evolutionary basis for pain recognition, or lack thereof, in others}

Detection of pain expression in others is thought to be an evolutionary advantage that alerts humans to potential physical threats. The close coupling of detection of pain with empathic reactions in the observer are thought to be adaptive and promote sustainability of the social group (40). However, it is also proposed that an 'evolutionary skepticism' (ie, bias to underestimate another person's pain), developed over human evolution to counterbalance the risk in initiating helping behaviours. Similarly, humans appear to have developed skills in detecting social cheating in others to protect against others 'faking' pain to take advantage for unrelated reasons. These factors may explain why people often seek corroborating contextual evidence such as visible signs of injury before accepting the pain cues of another as authentic. Although a tendency toward underestimation of another person's pain may have been adaptive in evolutionary terms, it can lead to mismanagement of pain in the context of modern clinical health care. Failure to account for interpersonal purposes and social consequences of pain expression and detection in clinical patient care (41) may partially explain the limited effectiveness and resistance to integration of standardized pain assessment tools in hospital settings.

\section{Motivation to act on pain detected in others}

Goubert et al (28) have proposed that empathy for pain can activate different affective responses, which motivate the observer to actions consistent with the affective state when pain is observed. If sympathetic affect is triggered, the observer will be oriented toward the person in pain, and their affective state will enable comforting and other supportive behaviours. However, if the observer's affective responses are oriented toward the self, then observing pain in another may trigger distress and anxiety, and behaviours that seek to distance oneself by withdrawing or denying the pain.

The ability to discriminate between the sense of knowing the other person's pain and one's own affective responses are considered fundamental to the delivery of effective care to people in pain. However, empathetic recognition of pain (or empathy in general) in health care professionals does not necessarily translate into better pain management in clinical settings (42). There may be other competing factors in the social milieu of the clinical setting that negate or moderate sympathetic motivation to act on pain signals from patients. For example, clinicians may have decreased motivation to detect pain in another because they become desensitized, they suppress their empathetic reactions, they may see other aspects of their clinical care as more important or there may be interpersonal power issues that override individual motivation to act to treat pain in patients $(42-44)$.

\section{Can pain recognition skills be taught and motivation to act enhanced?}

There is little direct evidence of the effectiveness of training individuals to improve their pain recognition skills. We found only two studies that directly investigated this in health care professionals. In one study (36), a $30 \mathrm{~min}$ training session with occupational and physical therapists improved the accuracy of pain facial expression recognition skills in adult patients. Another study (45) showed that nurses caring for ill infants could be trained to proficiently use a neonatal facial coding system to measure pain facial expression. These studies do not provide enough evidence to allow any general conclusions about the efficacy of such training for improving skills or outcomes. There is, nevertheless, indirect evidence from decades of research on pain assessment validation studies, in which researchers were trained in pain observation methods and achieved inter-rater reliability as well as concordance with other indicators of pain. Others (40) have suggested that feedback on accuracy of pain recognition and correction of systematic bias can improve pain detection skills. It remains to be tested whether the desensitization or suppression of pain helping behaviours described by health care professionals can be prevented or ameliorated with training or psychological therapy.

\section{DISCUSSION}

Our critical review of the literature found an insufficient body of properly constructed studies to allow a clear conclusion on the clinical effectiveness of standardized pain assessment tools in acute care pediatrics. In view of the ongoing poor compliance 
seen in the use of these tools in clinical practice, despite professional and accreditation mandates, we suggest that it is time to revisit the fundamental assumptions that underpin the view that they are good things to use. We are not criticizing the fundamental importance of assessment to the diagnosis and treatment of pain per se. However, the wholesale adoption of standardized pain assessment tools is not occurring, and it is difficult to justify continued costly efforts to implement and police them in the absence of research evidence of clear benefit. Implementation of standardized pain assessment tools may not be without risk. Adoption of clinical monitoring protocols can have negative unintended consequences (46) and can divert resources from delivery of therapies. Questions have been raised as to whether it contributes to overprescribing sedatives (47).

It remains unknown whether meaningful improvement in innate pain recognition skills can be achieved or sustained in health care professionals, if defects in pain recognition processing can be corrected or compensated, or if motivation to act on pain recognition can be improved. Provocatively, we must consider the possibility that humans are inherently flawed in the assessment of pain in others (vulnerable to underestimating or overestimating pain). If so, human-mediated pain assessment should be replaced by technology, as has been done with other vital signs (eg, temperature, blood pressure). An interesting study (48) found that a computer algorithm could discriminate, with 90\% accuracy, between facial expression in neonates photographed in pain and nonpain states. Shifting resources from trying to institutionalize standardized pain assessment tools by clinicians to a technological solution for detecting pain may free up resources for improving use of pain assessment information in decisions about management of pain.

Another approach would be to select the most sensitive observers with positive motivational attributes to act on their observations. For children, parents may, in many cases, have greater motivation than health care professionals for children to receive pain treatment, and may be more sensitive observers. Parents could play a greater role than currently allowed by health care services in their children's pain assessment and treatment.

We have argued in the present paper that one of the difficulties in implementing and sustaining the use of pain assessment tools in clinical practice is the lack of good-quality

\section{REFERENCES}

1. Howard R, Carter B, Curry J, et al. Good practice in postoperative and procedural pain management. Paediatr Anaesth 2008;18(Suppl 1):1-80.

2. Joint Commission on Accreditation of Healthcare Organizations. Improving the quality of pain management through measurement and action. <http://www.reliefinsite.com/downloads/Improving_the Quality_of_Pain_Mgmt_Thru_Measurement_and_Action_JCAHO. pdf $>$ (Version current at January 22, 2009).

3. Taylor EM, Boyer K, Campbell FA. Pain in hospitalized children: A prospective cross-sectional survey of pain prevalence, intensity, assessment and management in a Canadian pediatric teaching hospital. Pain Res Manage 2008;13:25-32.

4. Narasimhaswamy S, Vedi C, Xavier Y, et al. Effect of implementing pain management standards. J Gen Intern Med 2006;21:689-93.

5. Mularski RA, White C, Overbay D, et al. Measuring pain as the 5 th vital sign does not improve quality of pain management. J Gen Intern Med 2006;21:607-12.

6. Bucknall T, Manias E, Botti M. Nurses' reassessment of postoperative pain after analgesic administration. Clin J Pain 2007;23:1-7. evidence for efficacy, effectiveness or cost-benefit in relation to pediatric patient or process outcomes. We have explored some of the research that suggests that there may be greater variability than previously appreciated in the ability and motivation of the person assessing pain, which cannot be either improved or overcome by standardized methods. We support the view that further research is needed to understand the intra- and interpersonal dynamics in the clinical assessment of pain in children (49). Properly conducted randomized trials comparing structured pain assessment tools with clinical judgment, with or without parent collaboration, or with computer-based assessments, are needed to answer questions about effectiveness and cost-benefit.

Some may argue that there are aspects of health care that do not need to be subjected to RCTs to underpin widespread adoption (50). In fact, recent reviews point out the dearth of research indicating patient benefit to many highly valued monitoring methods in intensive care settings (51) or nursing record systems across multiple settings (52). However, these practices do not suffer the same problem with compliance as pain assessment and appear to be valued to the degree that sufficient resources are allocated to ensure their thorough implementation. Poor compliance with pain assessment guidelines may not simply be an issue of the 'research-to-practice gap', but it may indicate unspoken resistance to use of methods that are overly simplistic, burdensome to patients, often inaccurate and perhaps even disrespectful of clinical expertise and experience. Clinicians need - and deserve - to know whether using structured pain assessment tools truly results in better patient outcomes or improvement in pain care processes that, in turn, improve patient outcomes. Until this evidence is available, guidelines recommending standardized pain assessment must be clearly labelled as being based on principles or evidence from other fields of practice and avoid implying that they are 'evidence-based' standards.

ACKNOWLEDGEMENTS: The authors thank Ruth Muscat for her assistance with the literature search, participants at the 2008 International Forum on Paediatric Pain for their feedback on the ideas presented in this paper, the reviewers for their constructive comments and Annie Saunders for her assistance with manuscript preparation.
7. Cochrane Effective Practice and Organisation of Care Group. Data collection checklist. <http://epoc.cochrane.org/en/ handsearchers.html> (Version current at January 27, 2009).

8. Cohen LL, Lemanek K, Blount RL, et al. Evidence-based assessment of pediatric pain. J Pediatr Psychol 2008;33:939-55.

9. Stinson JN, Kavanagh T, Yamada J, et al. Systematic review of the psychometric properties, interpretability and feasibility of self-report pain intensity measures for use in clinical trials in children and adolescents. Pain 2006;125:143-57.

10. von Baeyer CL, Spagrud LJ. Systematic review of observational (behavioral) measures of pain for children and adolescents aged 3 to 18 years. Pain 2007;127:140-50.

11. Kaplan CP, Sison C, Platt SL. Does a pain scale improve pain assessment in the pediatric emergency department? Pediatr Emerg Care 2008;24:605-8.

12. Friedrichs JB, Young S, Gallagher D, et al. Where does it hurt? An interdisciplinary approach to improving the quality of pain assessment and management in the neonatal intensive care unit. Nurs Clin North Am 1995;30:143-59. 
13. Simons J, MacDonald LM. Changing practice: Implementing validated paediatric pain assessment tools. J Child Health Care 2006;10:160-76.

14. Stevens B. Development and testing of a pediatric pain management sheet. Pediatr Nurs 1990;16:543-8.

15. Franck LS, Allen A, Oulton K. Making pain assessment more accessible to children and parents: Can greater involvement improve the quality of care? Clin J Pain 2007;23:331-8.

16. Johnston CC, Gagnon A, Rennick J, et al. One-on-one coaching to improve pain assessment and management practices of pediatric nurses. J Pediatr Nurs 2007;22:467-78.

17. Vihunen R, Sihvonen M. [Use of Faces Scale in children's selfassessment of pain.] Hoitotiede 1998;10:123-33.

18. Boughton $\mathrm{K}$, Blower C, Chartrand C, et al. Impact of research on pediatric pain assessment and outcomes. Pediatr Nurs 1998;24:31-5, 62.

19. Buchanan L, Voigtman J, Mills H. Implementing the Agency for Health Care Policy and Research pain management pediatric guideline in a multicultural practice setting. J Nurs Care Qual 1997;11:23-35.

20. Ellis JA, McCleary L, Blouin R, et al. Implementing best practice pain management in a pediatric hospital. J Spec Pediatr Nurs 2007; 12:264-77.

21. Falanga I, Lafrenaye S, Mayer SK, et al. Management of acute pain in children: Safety and efficacy of a nurse-controlled algorithm for pain relief. Acute Pain 2006;8:45-54

22. Furdon SA, Eastman M, Benjamin K, et al. Outcome measures after standardized pain management strategies in postoperative patients in the neonatal intensive care unit. J Perinat Neonatal Nurs 1998; 12:58-69.

23. Hamers JP, Huijer Abu-Saad H, Geisler FE, et al. The effect of paracetamol, fentanyl, and systematic assessments on children's pain after tonsillectomy and adenoidectomy. J Perianesth Nurs 1999;14:357-66.

24. Treadwell MJ, Franck LS, Vichinsky E. Using quality improvement strategies to enhance pediatric pain assessment. Int J Qual Health Care 2002;14:39-47.

25. Joyce BA, Keck JF, Gerkensmeyer JE. Evaluating the implementation of a pain management flow sheet. J Pediatr Nurs 1999; 14:304-12.

26. Jordan-Marsh M, Hubbard J, Watson R, et al. The social ecology of changing pain management: Do I have to cry? J Pediatr Nurs 2004;19:193-203.

27. Oakes LL, Anghelescu DL, Windsor KB, et al. An institutional quality improvement initiative for pain management for pediatric cancer inpatients. J Pain Symptom Manage 2008;35:656-69.

28. Goubert L, Craig KD, Vervoort T, et al. Facing others in pain: The effects of empathy. Pain 2005;118:285-8.

29. Deyo KS, Prkachin KM, Mercer SR. Development of sensitivity to facial expression of pain. Pain 2004;107:16-21

30. Calder AJ, Keane J, Manly T, et al. Facial expression recognition across the adult life span. Neuropsychologia 2003;41:195-202.

31. Le GR, Mondloch CJ, Maurer D, et al. Expert face processing requires visual input to the right hemisphere during infancy. Nat Neurosci 2003;6:1108-12.

32. Danziger N, Prkachin KM, Willer JC. Is pain the price of empathy? The perception of others' pain in patients with congenital insensitivity to pain. Brain 2006;129:2494-507.
33. Richards A, French CC, Calder AJ, et al. Anxiety-related bias in the classification of emotionally ambiguous facial expressions. Emotion 2002;2:273-87.

34. Harmer CJ, Bhagwagar Z, Perrett DI, et al. Acute SSRI administration affects the processing of social cues in healthy volunteers. Neuropsychopharmacology 2003;28:148-52.

35. Frigerio E, Burt DM, Montagne B, et al. Facial affect perception in alcoholics. Psychiatry Res 2002;113:161-71.

36. Sullivan MJ, Martel MO, Tripp DA, et al. Catastrophic thinking and heightened perception of pain in others. Pain 2006;123:37-44.

37. Kappesser J, Williams AC. Pain and negative emotions in the face: Judgements by health care professionals. Pain 2002;99:197-206.

38. Balda RCX, Guinsburg R, de Almeida MFR, et al. The recognition of facial expression of pain in full-term newborn infants by parents and health professionals. Arch Pediatr Adolesc Med 2000;154:1009-16.

39. Pillai Riddell RR, Craig KD. Judgments of infant pain: The impact of caregiver identity and infant age. J Pediatr Psychol 2007;32:501-11.

40. Williams AC. Facial expression of pain: An evolutionary account Behav Brain Sci 2002;25:439-55.

41. Craig KD. Social communication of pain enhances protective functions: A comment on Deyo, Prkachin and Mercer (2004). Pain 2004;107:5-6.

42. Campbell-Yeo M, Latimer M, Johnston C. The empathetic response in nurses who treat pain: Concept analysis. J Adv Nurs 2008;61:711-9.

43. Lauzon Clabo LM. An ethnography of pain assessment and the role of social context on two postoperative units. J Adv Nurs 2008;61:531-9.

44. McGrath PJ, Unruh A. Neonatal pain in a social context. In: Anand KJ, Stevens BJ, McGrath PJ, eds. Pain in Neonates, 2nd edn. Philadelphia: Elsevier, 2000:237-50.

45. Grunau RVE, Oberlander TF, Holsti L. Bedside application of the Neonatal Facial Coding System in pain assessment of premature neonates. Pain 1998;76:277.

46. Adams BD, McHugh KJ, Bryson SA, et al. The law of unintended consequences: The Joint Commission regulations and the digital rectal examination. Ann Emerg Med 2008;51:197-201,201.e1.

47. Lucas CE, Vlahos AL, Ledgerwood AM. Kindness kills: The negative impact of pain as the fifth vital sign. J Am Coll Surg 2007;205:101-7.

48. Brahnam S, Chuang CF, Sexton RS, et al. Machine assessment of neonatal facial expressions of acute pain. Decis Support Syst 2007;43:1242-54.

49. Carter B. What pain assessment guidelines tell us and what they may miss. J Child Health Care 2008;12:170-2.

50. Glasziou P, Chalmers I, Rawlins M, et al. When are randomised trials unnecessary? Picking signal from noise. Br Med J 2007;334:349-51

51. Ospina-Tascon GA, Cordioli RL, Vincent JL. What type of monitoring has been shown to improve outcomes in acutely ill patients? Intensive Care Med 2008;34:800-20.

52. Currell R, Urquhart C. Nursing record systems: Effects on nursing practice and health care outcomes. Cochrane Database Syst Rev 2003;(3):CD002099. 


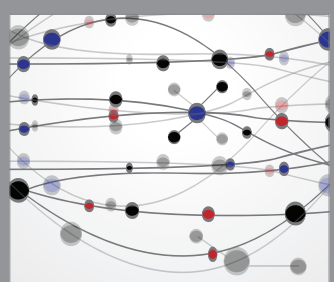

The Scientific World Journal
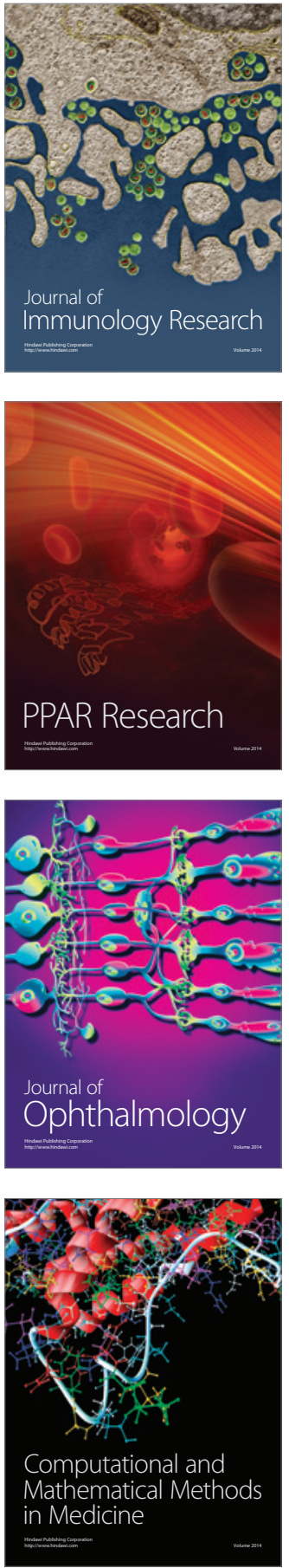

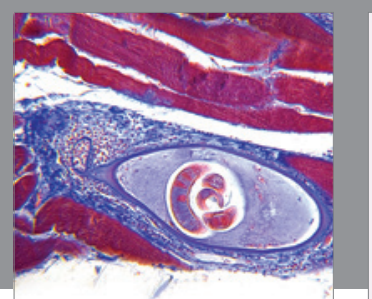

Gastroenterology Research and Practice

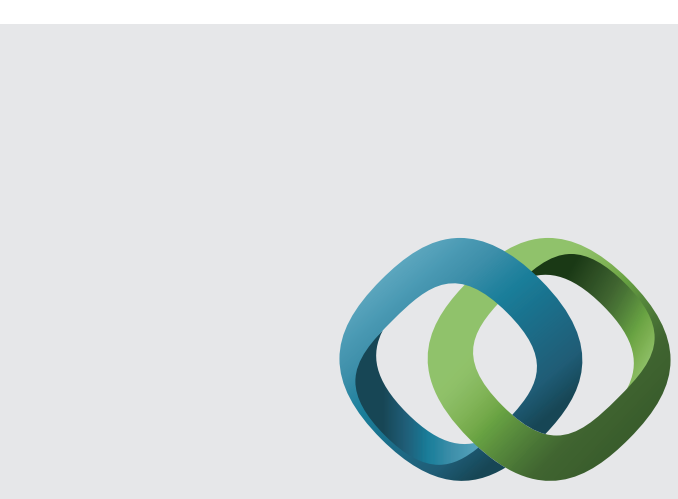

\section{Hindawi}

Submit your manuscripts at

http://www.hindawi.com
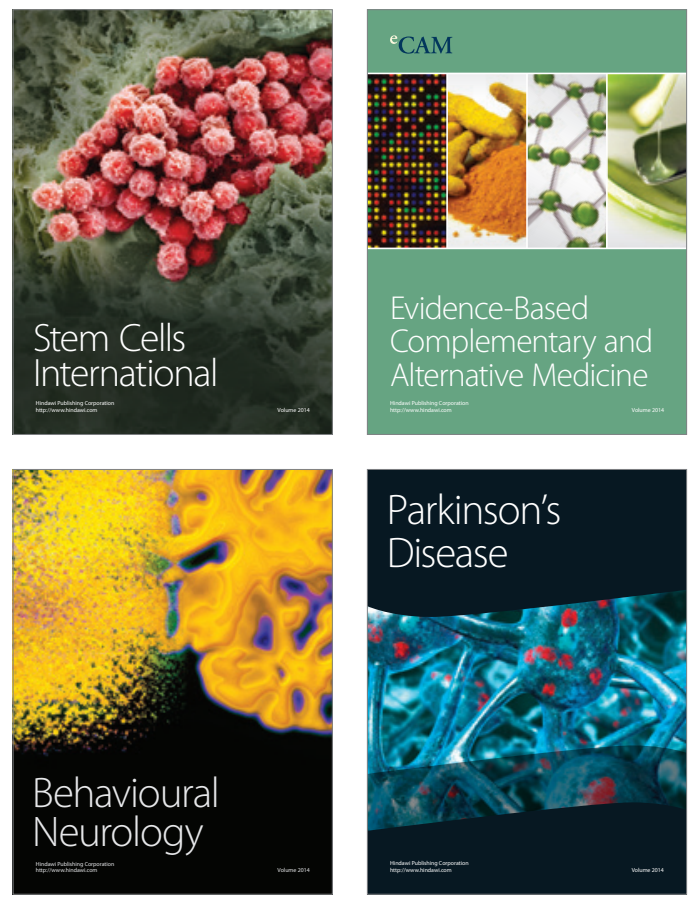
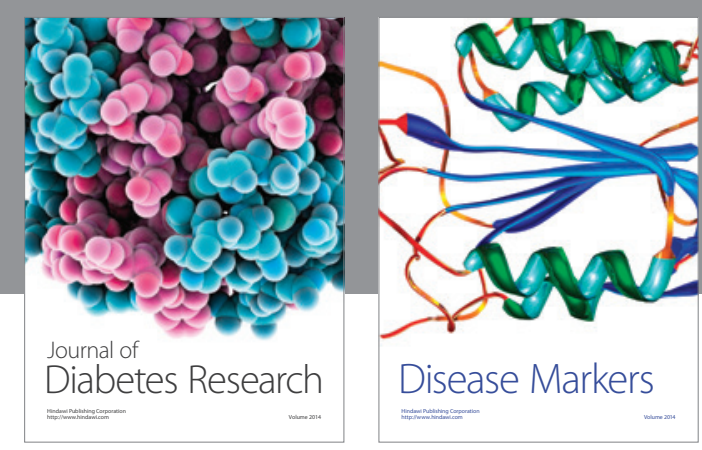

Disease Markers
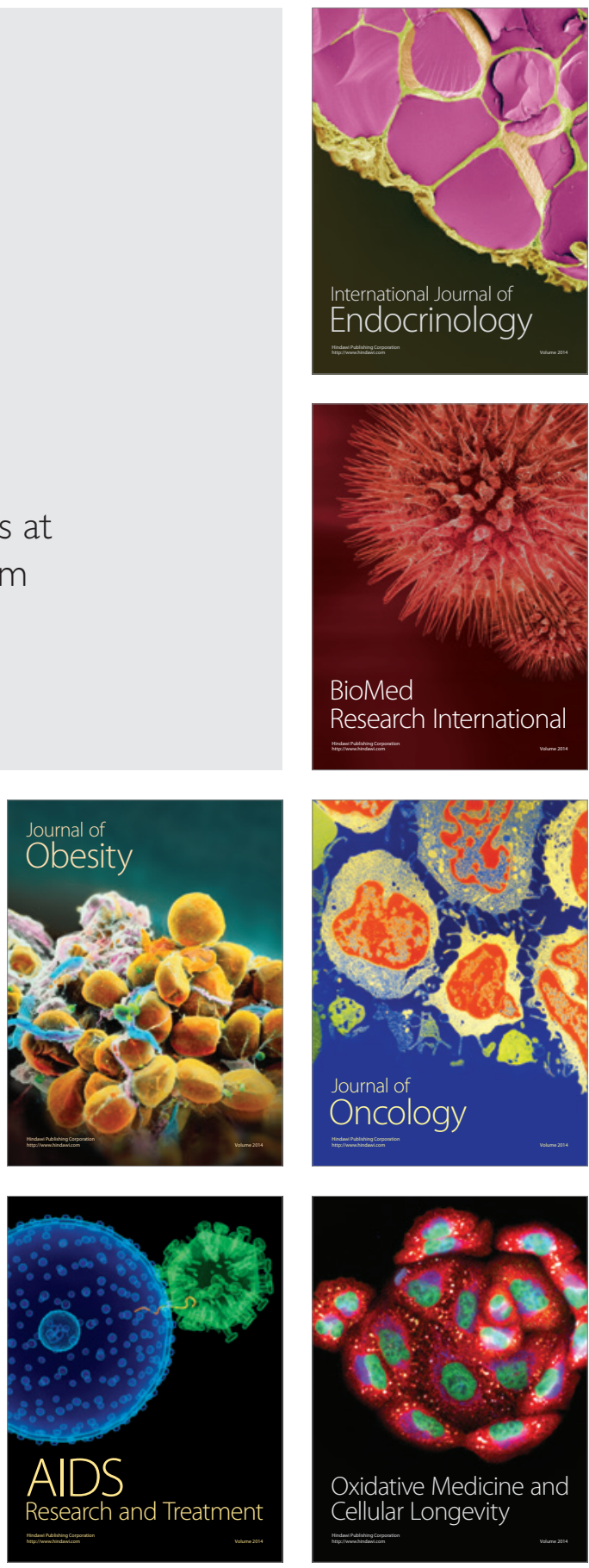